\title{
Design and Analysis of Cleaning Mechanism for an Intermittent Screw-Driven Pipeline Robot
}

\author{
Zhiqin Cai, Chao Lin and Dehong Huo
}

\begin{abstract}
For achieving the compact size and large traction force of cleaning robots, this paper presents an intermittentcleaning robot based on the screw-driven mechanism. The robot has two working modes: linear reciprocating cleaning (LRC) mode and spiral reciprocating cleaning (SRC) mode. The working principle, kinematic analysis, traction and driving force calculations for both working modes, were obtained and compared. Furthermore, in order to ensure the working stability, the failure mode analysis was performed. Simulation and experiments were conducted to verify the proposed robotic mechanism. The results show that the cleaning capabilities of the proposed intermittent-cleaning robots are superior to those standard screw-driven cleaning mechanisms.
\end{abstract}

Index Terms-Cleaning robot, intermittent screw-driven, traction force, failure mode, small pipeline.

\section{I.INTRODUCTION}

$\mathrm{I}$ N-PIPE cleaning robots are playing an increasingly prominent role in the area of pipeline cleaning which can replace labour workers. However, in-pipe robots would fail if they could not suffer the deterioration process of the pipeline walls during operation, as a result the working stability, security, and efficiency of pipelines would deteriorate. Therefore it is essential to improve the adaptability of the robots.

As one of the main components of cleaning robots, the movement pattern determines the cleaning capacity of the robots. According to Roh et al. [1], in-pipe robots can be classified into several specific types depending on their applications. For pipeline inspection, it can be mainly classified as wheel type, caterpillar type, inchworm type, wall-press type, walking type, and screw type [2]-[6]. In pipeline cleaning application, the robotic systems are required to provide enough traction force to against the obstacles, so PIG (Pipeline Inspection Gauge) and screw-driven robots are typically employed. PIG type is well-known as the most commercial method and can be applied in the cleaning of pipelines with large diameters [7]-[9]. However, this method is limited by the pipe diameter and can only move in the direction of the fluid flow. In order to extend the application scope, especially in the pipeline with small diameter $(20-50 \mathrm{~mm})$ and the drying condition required, the screw mechanisms offering the unique advantages, such as greater traction, more compact structure and better stability, could be widely employed [10]-[14]. A typical screw-driven robot is usually composed of a rotator, elastic support arms, rollers and a driving motor [15]. Although the carrying capacity is usually stronger than the other movement types, the traction is still not enough in the pipeline with small diameter due to the continuous motion feature and the limitation of motor power. Based on the screw-driven principle, this study proposes an intermittent-cleaning robot which can overcome these problems.

This paper is organized as follows. After introduction to the concept of intermittent-cleaning robots, two cleaning modes are proposed. Then some related comparison, including the kinematic analysis, traction calculation and driving torque, are obtained. In addition, a 3D design of the in-pipe robot platform is developed for the simulation. Finally, experiments are conducted to verify the model and simulation.

\section{II.CONCEPT OF THE CLEANING ROBOT}

A typical screw-driven robot is shown in Fig.1 (a), and the roller has a spiral angle $a$. When the rotation of the motor is clockwise or counter-clockwise, the whole body moves forward or backward respectively. However, this type of robot is only suited to the relatively light loading condition. For those high loading conditions, an intermittent screw drive mechanism should be used, as illustrated in Fig.1 (b).

Compared with the typical screw drive robots, an intermittent screw drive robot is composed of two lock-up mechanisms, rollers, a rotator and three motors (one is the main driving motor and the other two are the locking motors). Although the structure is more complex, the locking characteristic of this compact design will substantially improve the stability and carrying capacity.

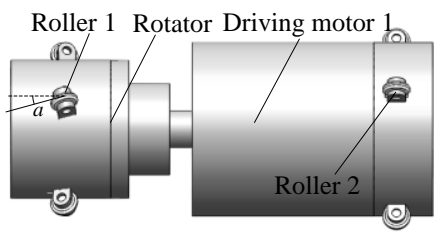

(a)

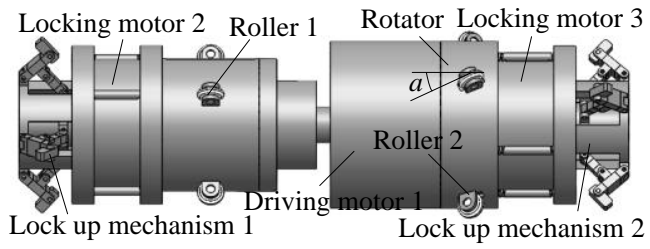

(b) 
Fig.1 Screw drive robots. (a) Typical screw drive robot. (b) Intermittent screw drive robot.

Based on the intermittent screw drive theory, a robot with cleaning capability is proposed as shown in Fig.2. The robot is composed of an intermittent screw mechanism and a cleaning mechanism, and the latter includes a pair of curve-face gears and hairbrushes. As one of the main components of the cleaning mechanism, the curve-face gear obeys the varying-spiral theory with variable hand of spiral, lead value and spiral angle, which can achieve the composited movement, i.e. the rotation of intersecting axles as well as the movement of output axle.

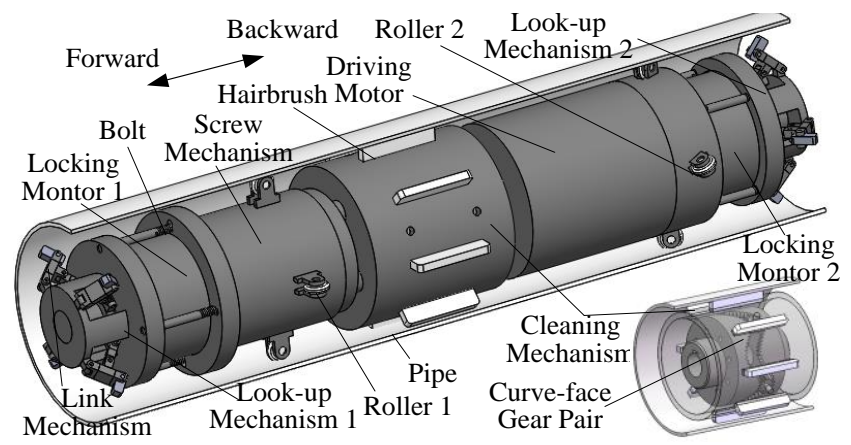

Fig. 2 The proposed cleaning robot.

The working principle of the proposed cleaning robot is explained as follows.

\section{1) Moving Forward - Step I}

The screw mechanism and locking mechanism 1 move forward together, while the driving motor turns in clockwise direction and locking mechanism 2 keeps locked due to the constraint of the link mechanism. Thus, the roller 1 acts as a guide during movement;

\section{2) Moving Forward - Step II}

The cleaning mechanism, driving motor and locking mechanism 2 move forward together, while the screw mechanism and locking mechanism keeps locked, the driving motor turns in counter-clockwise direction. Thus, the roller 2 acts as a guide during the movement.

\section{3) Cleaning Mode}

The cleaning mechanism keeps working whether the driving motor turns in clockwise or counter-clockwise direction, which can prevent the deterioration of the pipe wall roughness and the reduction of the internal diameter.

The working status of the three motors under these working conditions are summarised in Table I.

TABLE I.

SUMMARY OF MOVING MODE AND CLEANING MODES

\begin{tabular}{cccc}
\hline \hline & Motor 1 & Driving motor & Motor 2 \\
\hline move forward-step I & $\circ$ & $\cup$ & $\bullet$ \\
move forward-step II & $\bullet$ & $\cup$ & $\circ$ \\
cleaning mode & Null & $\cup / U$ & Null \\
\hline \hline
\end{tabular}

$\bullet$ Motor is active; oMotor is inactive; $\mathcal{U}$ or $\mathcal{U}$ is motor rotation directions. Null is motors have no effects.

Notably, the cleaning mechanism has two working modes depending on curve-face gears mounted in pairs either face-toface or back-to-back. One is linear reciprocating cleaning
(LRC) mode and another one is spiral reciprocating cleaning (SRC) mode.

\section{1) LRC Mode}

A pair of curve-face gears are fixedly installed back-to-back as shown in Fig.3 (a). Hairbrushes are pulled forward and backward by the axial movement of the cylindrical gear. The cleaning resistance depends on the surface roughness between the pipe wall and the hairbrush. In order to clean the whole internal surface of the pipe, the surface of cleaning mechanism should be covered with hairbrushes.

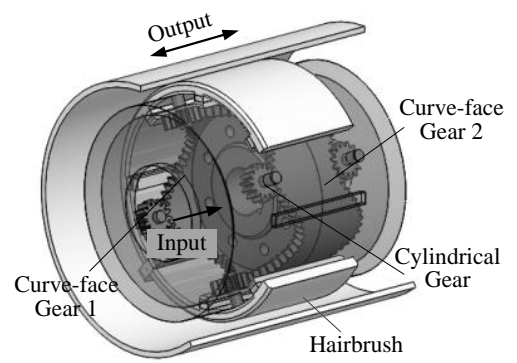

(a)

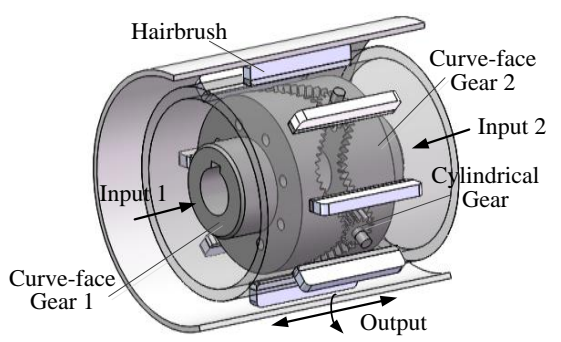

(b)

Fig. 3 two working modes. (a) LRC mode. (b) SRC mode.

\section{2) SRC Mode}

A pair of curve-face gears are installed face-to-face as shown in Fig. 3 (b). Hairbrushes move along the spiral trajectory of the cylindrical gear. Due to the motion characteristics of the hairbrushes, the number of hairbrushes is fewer than the LRC mode, which has less surface roughness requirement for the hairbrushes and pipeline wall. This mode can be regarded as a dual-input/single-output system, which is the same as the planetary differential gear train.

\section{III.KINEMATIC ANALYSIS FOR}

As the main part of the cleaning mechanism, curve-face gear pair's meshing trajectory follows a composite motion with rotation about its axis as well as reciprocal rectilinear motion. It can be defined as a varying-spiral movement with variable lead and spiral angle. Therefore, the motion rule of this gear pair can be explained by the spiral theory of meshing point $P$. 


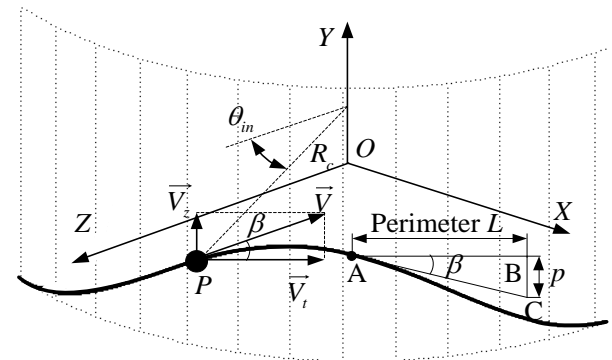

Fig.4. Kinematic analysis.

In order to obtain the basic parameters, the influence of traction is assumed to be negligible. When the nut moves along the pitch curve, the motion path is likely to be a spiral motion as shown in Fig.4. The cylindrical coordinates OXYZ is established as shown in Fig.4, where $\mathrm{Y}$ is axial direction of the cylinder. The motion path of cylindrical gear can be regarded as a spiral motion of point $P . \vec{V}$ is the tangent velocity of point $P$ along the spiral line.

The cleaning displacement can be defined as

$$
s=r(0)-r\left(\theta_{m}\right)
$$

where $r\left(\theta_{m}\right)$ is the pitch curve of non-cylindrical gear, $\theta_{m}$ is the rotation angle of non-cylindrical gear, which can be calculated by

$$
\theta_{m}=\arctan \left(C_{1} \tanh \left(C_{2} \theta_{i n}\right)\right)
$$

where $C_{1}=\left((k-1)(k+1)^{-1}\right)^{0.5}$ and $C_{2}=R_{c}\left(a\left(-1+k^{2}\right)^{0.5}\right)^{-1}$, which can be determined by the inverse function of $\theta_{i n}=\frac{1}{R} \int_{0}^{\theta_{m}} r(\theta) d \theta ; k$ is the eccentricity; $R_{c}$ is the radius of curve-face gear on OXZ plane; $a$ is the semi-axis length and $\theta_{i n}$ is the input angle.

The trajectory equation of point $P$ can be expressed as

$$
\left\{\begin{array}{c}
x=R_{c} \cos \left(\theta_{i n}\right) \\
y=r(0)-r\left(\theta_{m}\right) \\
z=R_{c} \sin \left(\theta_{i n}\right)
\end{array}\right.
$$

The tangent velocity $\vec{V}$ can be decomposed into axial velocity $\overrightarrow{V_{a}}$ and circular velocity $\vec{V}_{t}$, which can be calculated by

$$
|\vec{V}|=\left\{\begin{array}{l}
\left|\overrightarrow{V_{z}}\right|=\frac{d y}{d \theta_{i n}} \omega_{i n}=\frac{d y}{d \theta_{m}} \frac{d \theta_{m}}{d \theta_{i n}} \omega_{i n} \\
\left|\overrightarrow{V_{t}}\right|=R_{c} \omega_{i n}
\end{array}\right.
$$

where $\omega_{i n}$ is the input rotational speed .

The spiral characteristics are generally described by the lead and spiral angles. As a result, the movement characteristics can be extracted from these two parameters.

\section{1) Determination of the Lead}

The value of the lead $p\left(\theta_{i n}\right)$ can be calculated by

$$
p\left(\theta_{i n}\right)=\frac{2 \pi\left|\overrightarrow{V_{z}}\right|}{\omega_{i n}}=2 \pi \cdot \frac{d y}{d \theta_{m}} \frac{d \theta_{m}}{d \theta_{i n}}
$$

\section{2) Determination of the Spiral Angle}

The spiral angle $\beta\left(\theta_{i n}\right)$ can be calculated by

$$
\beta\left(\theta_{i n}\right)=\arctan \left(\frac{\left|\overrightarrow{V_{z}}\right|}{\left|\overrightarrow{V_{t}}\right|}\right)
$$

Substitute Eq. (4) into Eq.(6):

$$
p\left(\theta_{i n}\right)=2 \pi R_{c} \tan \beta\left(\theta_{i n}\right)
$$

Combining Eq.(4), Eq. (5) and Eq.(7), the movement speed can be written as

$$
\left|\overrightarrow{V_{z}}\right|=R_{c} \omega_{i n} \tan \beta\left(\theta_{i n}\right)
$$

From Eq.(4)-(6), the cleaning trajectory of the robot is a varying spiral movement with variable hand of spiral, lead and spiral angles, which can be obtained by

$$
\left\{\begin{array}{l}
p\left(\theta_{i n}\right)=2 \pi \cdot \frac{C_{3} C_{5} \sin 2 \theta_{m}}{\left(1-k \cos 2 \theta_{m}\right)^{2}} \frac{1-\tanh \left(C_{2} \theta_{i n}\right)^{2}}{1+C_{4} \tanh \left(C_{2} \theta_{i n}\right)^{2}} \\
\beta\left(\theta_{i n}\right)=\arctan \left(\frac{p\left(\theta_{i n}\right)}{2 \pi R_{c}}\right)
\end{array}\right.
$$

For simplicity, define $C_{3}=R_{c}(a(1+k))^{-1}, C_{5}=2 a k\left(1-k^{2}\right)$, $C_{4}=(k-1)(1+k)^{-1}$.

In the above equations, $\overrightarrow{V_{a}}, p\left(\theta_{i n}\right)$ and $\beta\left(\theta_{i n}\right)$ are simply the resolution under no-loading conditions. In fact, the loading (resistance) is a main factor affecting the actual working performance of the cleaning robot, and hence needs to be considered in the model. The improved expression of the movement velocity $\overrightarrow{V_{a}}$, from the viewpoint of the forces acting on the cylindrical gear, is derived as follows:

As can be seen in the Fig.5, $F_{a}, F_{n}, F_{t}$ and $f$ are axial resistant force, normal force, tangent force and frictional force, respectively. $T$ is the loading moment.

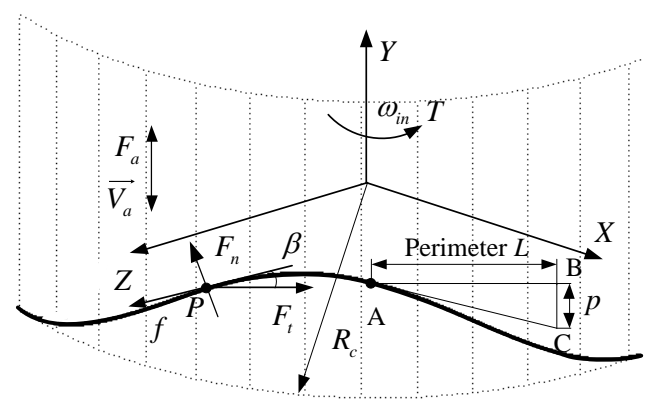

Fig.5. Force analysis.

According to the principle of spiral transmission, when the cylindrical gear is moving, the force condition of the curve-face gear can be expressed as

$$
\left\{\begin{array}{l}
F_{a}=F_{n} \cos \beta\left(\theta_{i n}\right)-f \sin \beta\left(\theta_{i n}\right) \\
T=F_{t} R_{c}=\left(F_{n} \sin \beta\left(\theta_{i n}\right)+f \cos \beta\left(\theta_{i n}\right)\right) R_{c} \\
f=u^{\prime \prime} F_{n}
\end{array}\right.
$$

where $u$ "is the frictional coefficient of curve-face gear pair.

From Eq.(10), the relation between axial resistant force $F_{\mathrm{a}}$ 
and traction torque $T$ is determined by the lead $p$, as follows:

$$
F_{a}\left(\theta_{i n}\right)=\frac{T\left(2 \pi R_{c}-u " p\left(\theta_{i n}\right)\right)}{R_{c}\left(p\left(\theta_{i n}\right)+u " 2 \pi R_{c}\right)}
$$

In a typical DC motor, the relation between the traction torque $T$ and the rotational velocity $\omega_{i n}$ follows a linear relationship [16]:

$$
\omega_{i n}=\omega_{0}\left(1-\frac{T}{T_{0}}\right)
$$

where $\omega_{0}$ is the no-loading rotational velocity and $T_{0}$ is the rated torque.

Combining Eq.(8) and Eq.(12), the movement speed can be written as:

$$
\left|\overrightarrow{V_{a}}\right|=\frac{p\left(\theta_{i n}\right) \omega_{0}}{2 \pi}\left[1-\frac{F_{a} R_{c}\left(p\left(\theta_{i n}\right)+2 \pi R_{c} u "\right)}{T_{0}\left(2 \pi R_{c}-u^{\prime \prime} p\left(\theta_{i n}\right)\right)}\right]
$$

In fact, Eq.(13) is a general formulation, that is , the actual movement speed can be expressed as:

$$
\left|\overrightarrow{V_{P}}\left(F_{C}, \theta_{i n}\right)\right|=\frac{p\left(\theta_{i n}\right) \omega_{0}}{2 \pi}\left(1-\frac{F_{C} R_{c}\left(p\left(\theta_{i n}\right)+2 \pi R_{c} u "\right)}{T_{0}\left(2 \pi R_{c}-u " p\left(\theta_{i n}\right)\right)}\right)
$$

where $F_{C}$ is the resultant force of cleaning mechanism along the axis.

For the cleaning mode, the output speed of the curve-face gear pair can be described as

$$
\left|\overrightarrow{V_{C}}\right|=\left\{\begin{array}{l}
\left|\overrightarrow{V_{P}}\left(F_{C 1}, \theta_{i n}\right)\right|(\text { LRC mode }) \\
\left|\overrightarrow{V_{P}}\left(F_{C 2}, \theta_{i n}\right)\right| / \sin \beta\left(\theta_{i n}\right) \text { (SRC mode) }
\end{array}\right.
$$

where $F_{C i}(\mathrm{i}=1,2)$ is the resultant force along the axis of two cleaning modes.

\section{IV.TRACTION ANALYSIS}

Based on the analysis above, the resistance will have a negative influence on the moving speed. In fact, due to the deterioration process, the diameter of some sections of the local pipeline may be significantly reduced. These sections of the pipeline produce large resistance, and hence are prone to produce blocking.

For the further analysis, it is assumed that (1) The interior shape of pipe is straight and circular; (2) The surface of the deteriorated wall is still circular; (3) The cleaning mechanism is a multi-body unit, where the internal part is a cylindrical rigid body and the external is an elastic body of torus shape; (4) The hairbrushes are described as a homogeneous and symmetric elastomer. The stress and deformation analysis of the unit is shown in Fig.6.

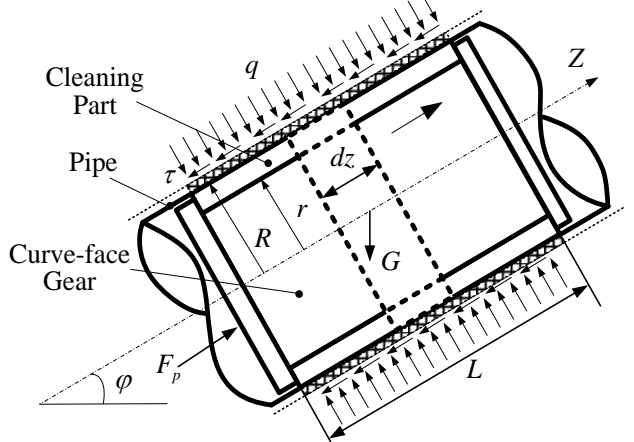

(a)

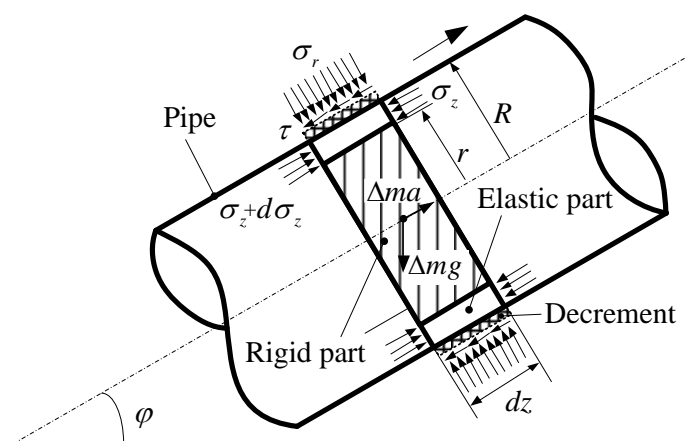

(b)

Fig.6. Stress and deformation analysis. (a) The forces acting on the cleaning mechanism. (b) The forces acting on the infinitesimal body.

The forces acting on the cleaning mechanism include gravity of the cleaning mechanism, the elastic pressure, and the friction force. As shown in Fig.6(a), where $R$ is the theoretical radius of pipeline; $r$ is the inner radius of elastic part; $L$ is the length of cleaning structure; $q$ is the radial stress due to the elastic deformation; $\tau$ is the frictional force of unit area; $F_{a}$ is the traction force; $\sigma_{r}$ and $\sigma_{z}$ are elastic force of unit area along radius and axial direction, respectively; $G$ is the gravity. Take the infinitesimal body $d z$ study object as shown in Fig.6 (b). In the cleaning process, the structure is in the equilibrium state, so does the infinitesimal body (as shown in Fig.6 (b)). $\varphi$ is the inclined angle, The equilibrium equation is:

$$
\pi\left(R^{2}-r^{2}\right) d \sigma_{z}+2 \pi \zeta R \tau d z-\Delta m g \sin \varphi=\Delta m a
$$

where $\tau=-u \sigma_{r}, \Delta m=\pi R^{2} \rho d z ; u$ is frictional coefficient between cleaning mechanism and pipeline.

$\zeta$ is the hairbrush area ratio, while in LRC mode $\zeta=1$ and in SRC mode the value of $\zeta$ depends on the number of hairbrush. $a$ is the acceleration under non-loading, which can be expressed as:

$$
a=\frac{d\left|\vec{V}_{z}\right|}{d \theta_{i n}} \frac{d \theta_{i n}}{d t}
$$

After rearrangement, the above equation can be rewritten as: $\pi\left(R^{2}-r^{2}\right) d \sigma_{z}-\left[2 \pi \zeta u R \sigma_{r}+\pi R^{2} \rho(a+g \sin \varphi)\right] d z=0$

The mathematical description of the $\sigma_{r}$ and $\sigma_{\theta}$ in Eq.(17) depends on the assumptions (4), the relation between $\sigma_{r}$ and 
$\sigma_{\theta}$ is determined by axial deformation $\sigma_{z}$, as follows[16]:

$$
\sigma_{r}=\sigma_{\theta}=\frac{1}{1-v}\left(\frac{\Delta E}{R}+v \sigma_{z}\right)
$$

where $\Delta$ is radial compression. $E$ is elasticity modulus. $v$ is Poisson's ratio.

Using equations (17) and (18), the equilibrium equation of the cleaning mechanism in the infinitesimal body $d z$ as shown in Fig.6 (b) can be expressed as

$$
\frac{\pi\left(R^{2}-r^{2}\right) d \sigma_{z}}{\frac{2 \pi \zeta u R}{1-v}\left(\frac{\Delta E}{R}+v \sigma_{z}\right)+\pi R^{2} \rho(a+g \sin \varphi)}=d z
$$

The general solution of Eq.(19) is

$$
\begin{array}{r}
\ln \left(\frac{2 \zeta u R}{1-v}\left(\frac{\Delta E}{R}+v \sigma_{z}\right)+R^{2} \rho(a+g \sin \varphi)\right) \\
=\frac{2 \zeta u R v}{\left(R^{2}-r^{2}\right)(1-v)} z+C
\end{array}
$$

where

$$
C=\left(\frac{2 \zeta u R \Delta E}{(1-v) R}+R^{2} \rho(a+g \sin \varphi)\right)
$$

Ultimately, the equilibrium equation when it passes through the length $L$ of pipeline as shown in Fig.6 (a) can be obtained by:

$$
\begin{array}{r}
\sigma_{z}=\frac{1-v}{2 \zeta u R v}\left(\frac{2 \zeta u R \Delta E}{(1-v) R}+R^{2} \rho(a+g \sin \varphi)\right) \\
\cdot\left(\exp \left(\frac{2 u \zeta R v L}{\pi\left(R^{2}-r^{2}\right)(1-v)}\right)-1\right)
\end{array}
$$

Hence, traction force $F_{p}$ acting on the cleaning mechanism is given:

$$
\begin{aligned}
F_{P}=\frac{\pi\left(R^{2}-r^{2}\right)(1-v)}{2 \zeta u R v} & \left(\frac{2 \zeta u R \Delta E}{(1-v) R}+R^{2} \rho(a+g \sin \varphi)\right) \\
& \left(\exp \left(\frac{2 u \zeta R v L}{\pi\left(R^{2}-r^{2}\right)(1-v)}\right)-1\right)
\end{aligned}
$$

For the cleaning mechanism, the traction force $F_{\mathrm{C}}$ can be described as

$$
F_{C}=\left\{\begin{array}{l}
F_{P}\left(\theta_{i n}\right)(\text { LRC mode) } \\
F_{P 1}\left(\theta_{i n 1}\right)-F_{P 2}\left(\theta_{i n 2}\right) \text { (SRC mode) }
\end{array}\right.
$$

where $F_{P n}(\mathrm{n}=1,2)$ is the traction which can be calculated by the Eq.(22).

Furthermore, the summation of the traction torque $T$ can be rewritten as follows:

$$
T \geq\left\{\begin{array}{l}
\frac{p\left(\theta_{i n 1}\right)}{2 \pi} F_{P}\left(\theta_{i n}\right)(\text { LRC mode }) \\
\frac{p\left(\theta_{i n 1}\right)}{2 \pi}\left(F_{P 1}\left(\theta_{i n 1}\right)-F_{P 2}\left(\theta_{i n 2}\right)\right)(\text { SRC mode })
\end{array}\right.
$$

\section{V.EXAMPLES AND ANALYSIS}

\begin{tabular}{|c|c|c|c|}
\hline Mechanism & Parameters & Symbol & Value \\
\hline \multirow{3}{*}{$\begin{array}{l}\text { Driving } \\
\text { motor }\end{array}$} & Revolving speed & $n$ & 120rpm \\
\hline & Torque & $T_{0}$ & $1,000 \mathrm{Nmm}$ \\
\hline & Eccentricity of curve-face gear & $k$ & 0.3 \\
\hline \multirow{3}{*}{$\begin{array}{l}\text { Curve-face } \\
\text { gear }\end{array}$} & Semi major axis of ellipse & $a$ & $17.2 \mathrm{~mm}$ \\
\hline & Radius of curve-face gear & $R_{c}$ & $32.8 \mathrm{~mm}$ \\
\hline & $\begin{array}{l}\text { Frictional coefficient of } \\
\text { curve-face gear pair }\end{array}$ & $u "$ & 0.1 \\
\hline \multirow[t]{4}{*}{ Pipe } & Radius of the pipe & $R$ & $40 \mathrm{~mm}$ \\
\hline & Inner radius of elastic body & $r$ & $30 \mathrm{~mm}$ \\
\hline & Density of cleaning mechanism & $p$ & $7.85 \mathrm{~g} / \mathrm{cm} 3$ \\
\hline & $\begin{array}{l}\text { Frictional coefficient between } \\
\text { cleaning } \\
\text { mechanism and pipeline }\end{array}$ & $u$ & 0.5 \\
\hline \multirow{5}{*}{$\begin{array}{l}\text { Cleaning } \\
\text { mechanism }\end{array}$} & Elasticity modulus & E & $7.8 \mathrm{Mpa}$ \\
\hline & Poisson's ratio & $v$ & 0.47 \\
\hline & Radial compression value & $\Delta$ & $\begin{array}{l}0.3 \mathrm{~mm}(1 \%- \\
2 \% R)\end{array}$ \\
\hline & hairbrush area ratio & $\zeta$ & $\begin{array}{l}0.15(\mathrm{LRC}) / \\
1(\mathrm{RSC})\end{array}$ \\
\hline & Length of pipeline & $L$ & $5 \mathrm{~mm}$ \\
\hline
\end{tabular}

The basic parameters of the cleaning robot will be obtained based on the proposed strategy. In the simulation, the robot moves forward in a horizontal pipe for a distance of $3,000 \mathrm{~mm}$, the cleaning time is limited within $30 \mathrm{~min}$, the radius of pipeline is $20 \mathrm{~mm}$ and the locking force is limited in $100 \mathrm{~N}$. According to above requirements, the other parameters of the robot and the pipe environment are given in Table II, and the related parameters are computed by using MATLAB.

TABLE II.

THE SIMULATION PARAMETERS

\section{A. Kinematic Analysis}

The simulation is consisted of two cleaning model (LRC model and SRC model), and the results are shown in Figs.7-8. In the LRC model, the cleaning trajectory obeys the law of linear reciprocating motion, while the SRC model presents the spiral reciprocating motion, as shown in Fig.7 (a). Both maximum axial displacements are approximately equal to $3 \mathrm{~mm}$.

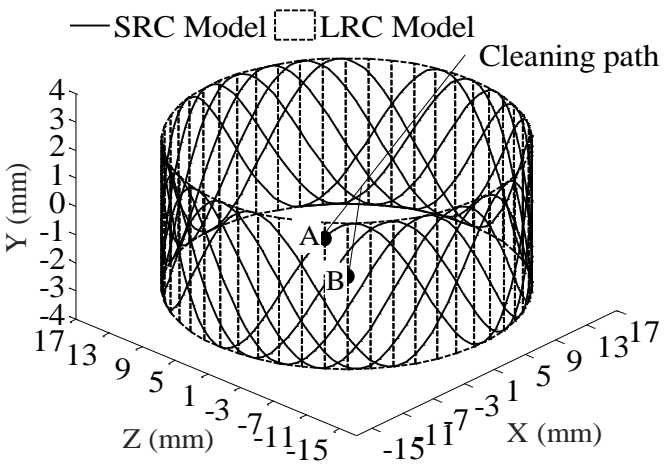

(a)

The cleaning velocity varies with the working time due to the time-varying spiral lead, as shown in Fig.7 (b). Because of the reciprocating cycles, the cleaning path of SRC model is longer than that in the LRC model at the same cleaning speed, which means the cleaning efficiency of the LRC model is much higher than SRC model. However, the traction force or torque (as 
shown in Fig.8) of LRC model is much larger than SRC model, which is mainly due to the larger hairbrush area ratio. In order to clean up completely, the hairbrush should completely cover the cleaning mechanism in LRC model, which increases the frictional force between cleaning mechanism and pipeline, and hence it needs a larger traction force.

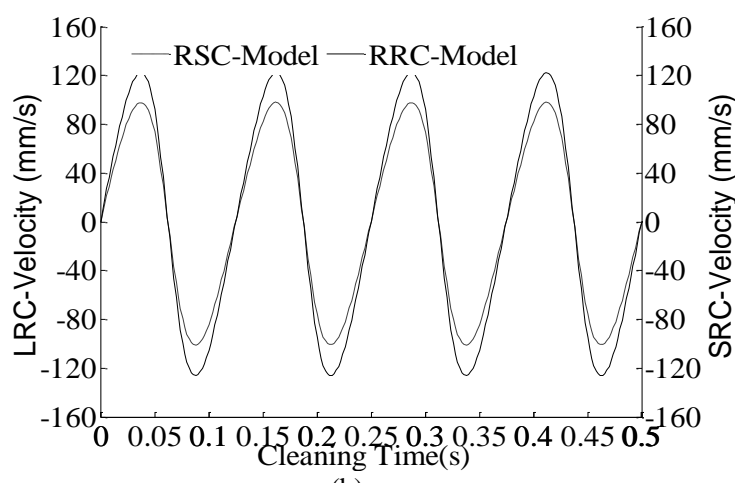

(b)

Fig.7. Comparison of kinematics between two cleaning models. (a) Cleaning path. (b) Cleaning speed.

Due to the smaller hairbrush area ratio, SRC model can drastically reduce the resistance as shown in Fig.8, the maximum cleaning force $F_{\mathrm{C}}$ in SRC model is only $11.2 \mathrm{~N}$ and maximum driving torque $T_{\mathrm{N}}$ is approximately 100 N.mm. Therefore, although the cleaning efficiency of SRC is much lower than LRC model, it can still meet the requirement, and its cleaning quality is the best in all existing cleaning mechanisms due to the spiral cleaning trajectory.

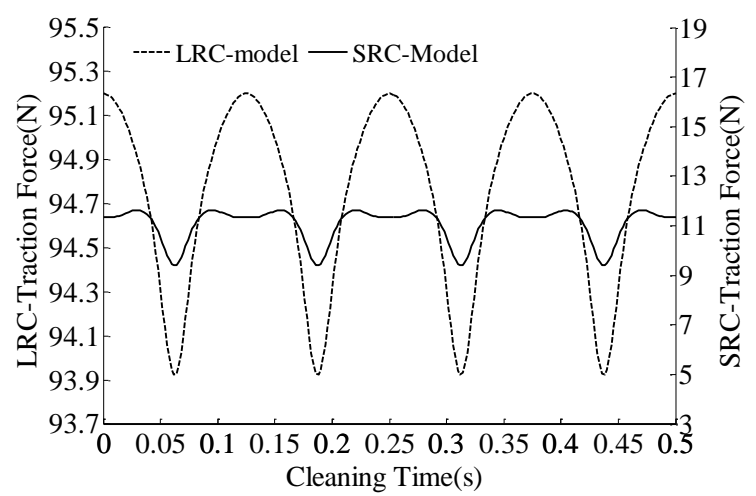

(a)

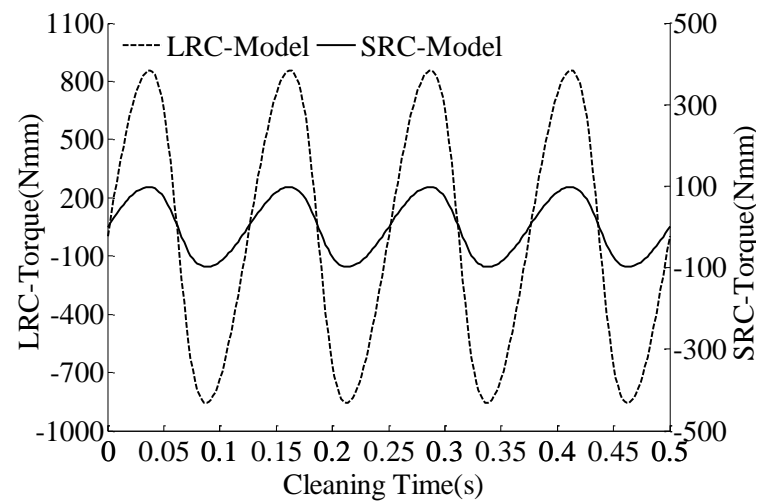

(b)
Fig.8. Comparison of traction between two cleaning models. (a)Traction force. (b) Traction torque.

\section{B. Failure Mode Analysis}

As above analysis, both cleaning models have advantages and disadvantages. However, the deterioration process of the pipeline walls during operation is uncertain, which results in the changes of frictional coefficient $u$, radial compression $\Delta$ and slant angle, etc. In order to ensure the working stability, the failure mode analysis is performed. According to the working conditions, this can be considered by three cases.

Case 1: The maximum value of frictional coefficient $u$. When the maximum torque $T_{0}$ is limited to $1000 \mathrm{Nmm}$, the maximum locking force is limited to $100 \mathrm{~N}$, radial compression $\Delta$ is limited to $0.3 \mathrm{~mm}$ ( $1 \%$ to $2 \%$ of pipeline radius), and the slant angel is zero.

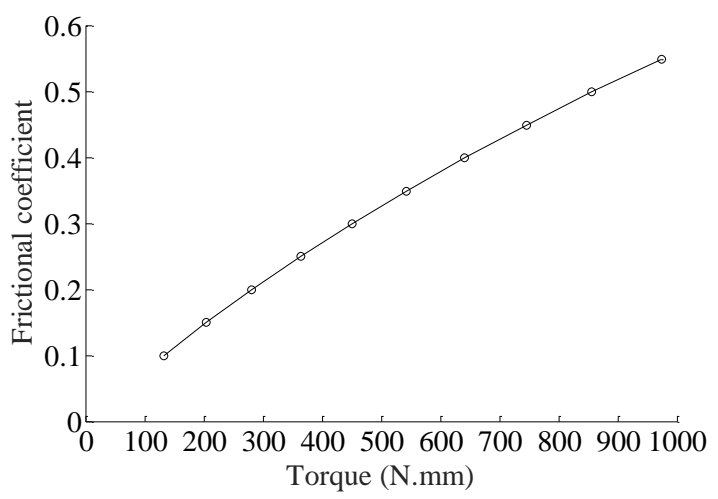

(a)

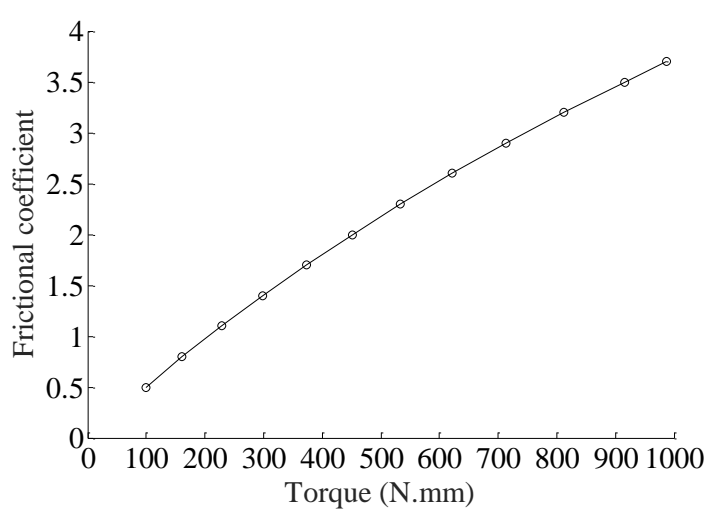

(b)

Fig.9. Comparison of frictional coefficient between two cleaning models. (a)LRC model. (b) SRC model.

As shown in Fig.9, during LRC model, the maximum allowable frictional coefficient is almost equal to 0.55 . This means that the allowable frictional coefficient of LRC model can arrange from 0 to 0.55 , while the frictional coefficient of $\mathrm{SRC}$ model varies from 0 to 3.7. It proves the better working stability of SRC model. According to some literature [11]-[12], the SRC model is more suitable for the flexible cleaning situation where the resistance is much heavier, whilst the LRC model is more suitable for dry friction situation where the resistance is lighter.

Case 2: The maximum value radial compression $\Delta$. When the torque $T_{0}$ is set to $1000 \mathrm{Nmm}$ as a constant, the maximum 
locking force is limited to $100 \mathrm{~N}$ and the slant angel is set to zero, the relationship between frictional coefficient $u$ and radial compression $\Delta$ can be described in Fig. 10.

Fig. 10 shows the different radial compression $\Delta$ in variable frictional coefficient $u$. The value of radial compression $\Delta$ decreases with the increase of the frictional coefficient $u$. Moreover, compared with LRC model, the cleaning quality of SRC model is better.

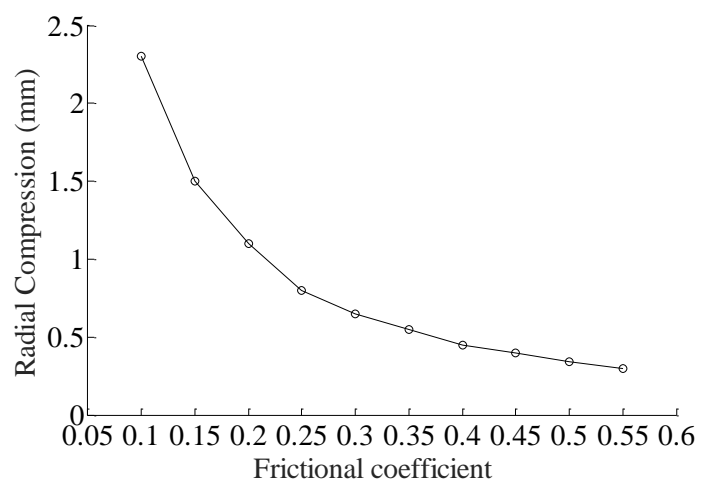

(a)

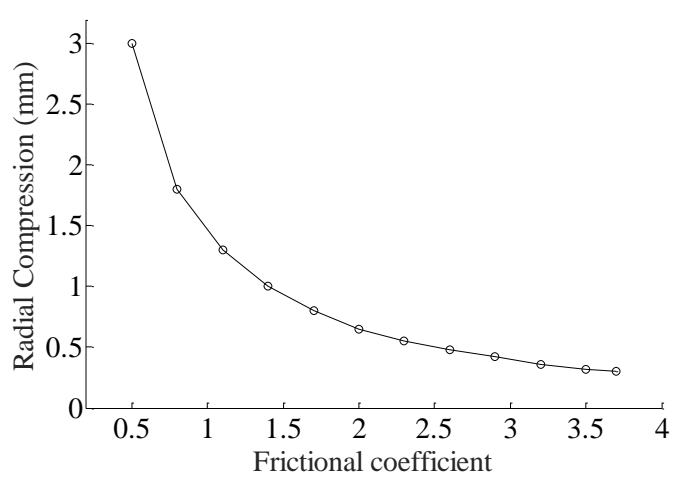

(b)

Fig.10. Comparison of radial compression between two models. (a)LRC model. (b) SRC model.

Case 3: The maximum value of slant angle. When the torque $T_{0}$ is set to $1000 \mathrm{Nmm}$ as a constant, the maximum locking force is limited to $100 \mathrm{~N}$. Since the maximum gravity is almost $7.6 \mathrm{~N}$, which will increase the locking force, so the relationship of gravity and traction force should be considered.

For LRC model, when the radial compression is less than $0.3 \mathrm{~mm}$ and the slant angle is zero, the traction force nearly reaches the limited locking force $100 \mathrm{~N}$. It proves that the LRC model is suit for the small slant angle $\left(-5^{\circ}\right.$ to $\left.5^{\circ}\right)$.

For SRC model, as shown in Fig.9 (b) and Fig.10 (b), the radial compression ranges from 0.3 to $3 \mathrm{~mm}$, while the frictional coefficient can vary from 0.5 to 3.7 . Moreover, the locking force is determined by the hairbrush area ratio.

In order to facilitate comparison, assume the slant angle is $90^{\circ}$, then the SRC model can be considered as two situations.

(1) As shown in Fig.11 (a), when radial compression is $0.3 \mathrm{~mm}$, the maximum frictional coefficient is 3.62 , it proves that the SRC model can suit any slant angels when radial compression is limited to $0.3 \mathrm{~mm}$. Moreover, the maximum frictional coefficient increases with the radial compression raises, which means that when the radial compression is over $0.3 \mathrm{~mm}$, the SRC model can't suit every slant angle any more.

(2) The hairbrush area ratio changes from 0.15 to 1 as shown in Fig11.(b): When the minimum radial compression is limited to $0.3 \mathrm{~mm}$, the frictional coefficient deceases with the increase of the hairbrush area ratio; Similarly, when the minimum frictional coefficient is limited to 0.5 , the radial compression reduces with the hairbrush area ratio raises.

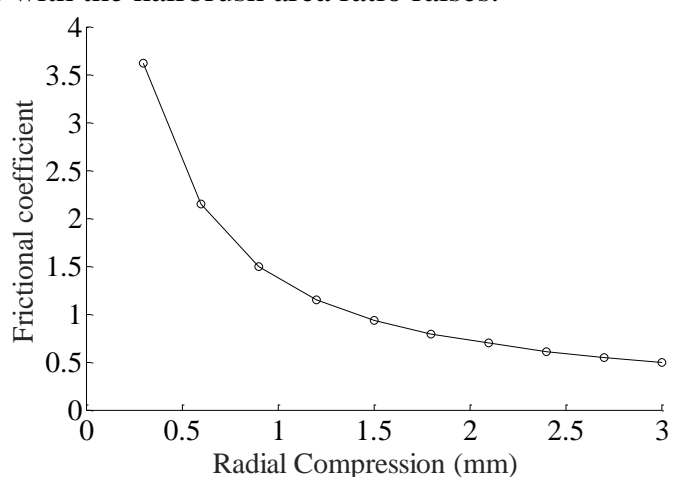

(a)

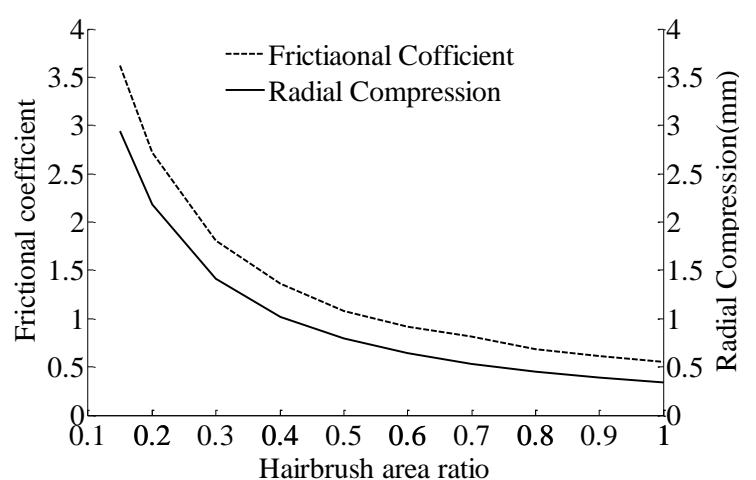

(b)

Fig.11.Failure model of SRC model. (a) Case 1. (b) Case 2.

\section{VI.EXPERIMENTAL VERIFICATION}

To validate the performance of the robot, a prototyped test bed was built and tested based on the simplified LRC model as shown in Fig.12. It should be noted that all the disturbing factors were not considered in the tests.

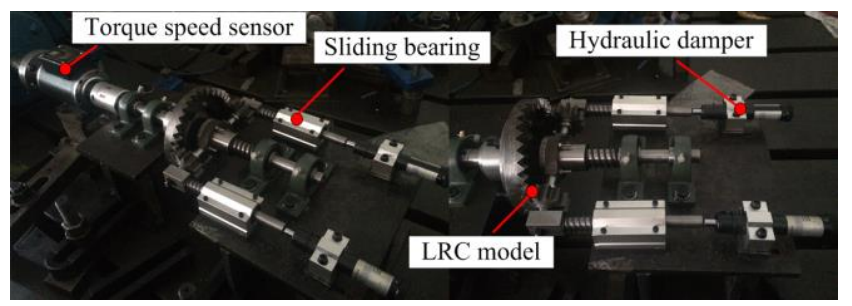

Fig.12.The test bed of the cleaning robot based on LRC model.

In order to reduce the influence of manufacturing errors, the size of cleaning mechanism is made twice larger than that in the simulation model. The input rotational speed is set to be $120 \mathrm{rpm}$, and the rated power is $3 \mathrm{~kW}$. The frictional force between pipeline wall and hairbrush was generated by the 
damper, as shown in Fig.12 (b).

Using equations (19) and (22), the value of the damping force $F_{\mathrm{D}}$ can be calculated, which is determined by the frictional coefficient $u$ and radial compression $\Delta$ and can be obtained as

$$
F_{D}=\frac{u-\left(0.213 \Delta^{2}-0.424 \Delta+0.256\right)}{0.036 \Delta^{2}-0.066 \Delta+0.048}
$$

Above Equation can be used to estimate the value of damping force $F_{\mathrm{D}}$ by changing the value of the radial compression $\Delta$ and the frictional coefficient $u$ of LRC model.

\section{A. Traction verification}

When the input velocity is constant, different traction forces $F_{C}^{\prime}$ can be obtained with the damping forces change, which can be calculated as follows:

$$
F_{C}^{\prime}=\frac{T_{0}^{\prime}}{2 R_{c}} \tan \alpha
$$

where $\alpha$ is the pressure angle, which can be obtained through the literature[17]. $T_{0}^{\prime}$ is traction torque which can be measured by the speed-torque sensor as shown in Fig.14(b).

Some essential results are shown in Figs.13. During the experimental time, the measured axial velocity and traction force $F_{C}^{\prime}$ are in good agreement with the theory analysis of the models.

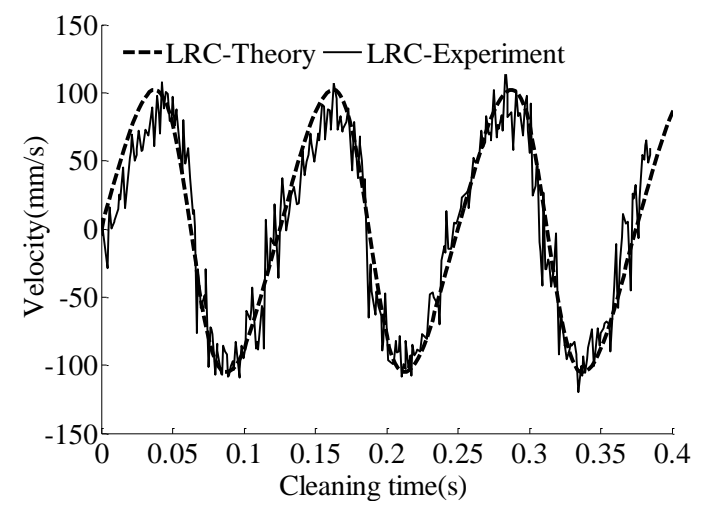

(a)

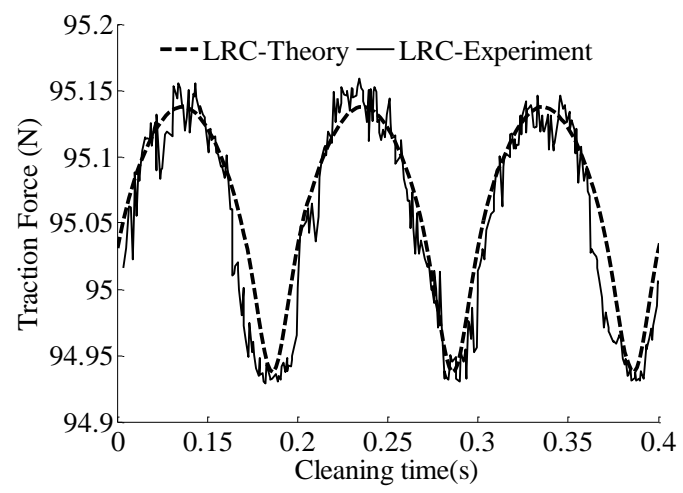

(b)

Fig.13.Comparison of simulation and theoretical results. (a) Cleaning speed. (b) Traction force.

\section{B. Failure Mode verification}

To validate the failure mode of the robot, the traction torque to be measured as the evaluating indicator. For the above two failure modes, Case 1 and Case 2 can be verified by the experimental results.

TABLE III.

THE COMPARISON OF CASE 1

\begin{tabular}{cccc}
\hline \hline $\begin{array}{c}\text { Frictional } \\
\text { coefficient }\end{array}$ & $\begin{array}{c}\text { Theoretical } \\
\text { torque }\end{array}$ & $\begin{array}{c}\text { Experimental } \\
\text { torque }\end{array}$ & $\begin{array}{c}\text { Relative } \\
\text { Error }\end{array}$ \\
\hline 0.1 & 132.2 & 140.8 & $6.0 \%$ \\
0.2 & 281.6 & 290.6 & $3.2 \%$ \\
0.3 & 450.5 & 465.9 & $3.3 \%$ \\
0.4 & 641 & 658.1 & $2.6 \%$ \\
0.55 & 998.5 & 1025.5 & $2.7 \%$ \\
\hline \hline
\end{tabular}

Case 1 verification: when radial compression is limited to $0.3 \mathrm{~mm}$ (the minimum radial compression of LRC model), the frictional coefficient varies from 0.1 to 0.55 (as shown in Fig.9 (a)), different traction torques can be obtained with the damping force changes. The comparison of the traction torque between theoretical and experimental results are shown in Table III. The relative error is suitable for evaluating the validity of the theoretical model.

TABLE IV.

THE COMPARISON OF CASE 2

\begin{tabular}{ccccc}
\hline \hline $\begin{array}{c}\text { Frictional } \\
\text { coefficient }\end{array}$ & $\begin{array}{c}\text { Radial } \\
\text { compression }\end{array}$ & $\begin{array}{c}\text { Theoretical } \\
\text { torque }\end{array}$ & $\begin{array}{c}\text { Experimental } \\
\text { torque }\end{array}$ & $\begin{array}{c}\text { Relative } \\
\text { Error }\end{array}$ \\
\hline 0.1 & 2.3 & 1000.5 & 979.7 & $2.1 \%$ \\
0.2 & 1.1 & 998.9 & 967.6 & $3.1 \%$ \\
0.3 & 0.65 & 1001.2 & 1051.2 & $5.0 \%$ \\
0.4 & 0.45 & 997.3 & 1027.8 & $3.0 \%$ \\
0.55 & 0.3 & 998.2 & 952.4 & $4.6 \%$ \\
\hline \hline
\end{tabular}

Case 2 verification: when the torque $T_{0}$ is set to $1000 \mathrm{Nmm}$, the frictional coefficient varies from 0.1 to 0.55 and the radial compression varies from $0.3 \mathrm{~mm}$ to $2.3 \mathrm{~mm}$. The comparison of the traction torque between theoretical (1000Nmm) and experimental results are as shown in Table IV. It is reasonable to conclude that the experimental verification presented is capable of the theory.

\section{VII.CONCLUSIONS}

For achieving the compact size and large traction force of cleaning robots, design, kinematic analysis, traction analysis and experimental evaluation of two new types cleaning model (LRC model and SRC model) based on intermittent screw mechanism are presented. The failure mode of various resistances caused by different frictional coefficients, radial compression, slant angles and hairbrush area ratios are discussed, which provides a guideline for designing such cleaning robots. Thus, optimal cleaning schemes can be obtained to best satisfy the requirements.

It is shown by the theoretical velocity analysis based on the traction force that no matter which models are used (LRC or SRC model), the cleaning capabilities of the proposed cleaning robot are higher than the typical cleaning mechanisms due to the reciprocating characteristics. 


\section{ACKNOWLEDGEMENT}

The authors would like to appreciate their supports from the National Natural Science Foundation of China (51275537) and Graduate Student Research Innovation Project (CYB15019).

\section{REFERENCES}

[1] S.G. Roh and H.R. Choi, "Differential-drive in-pipe robot for moving inside urban gas pipelines", IEEE Trans. Robot., vol. 21, no. 1, pp. 1-17, 2005.

[2] T. Oya and T. Okada, "Development of a steerable, wheel-type, in-pipe robot and its path planning", Advanced Robotics, vol. 19, no. 6, pp. 635$650,2005$.

[3] Y. Zhang and G. Yan, "In-pipe inspection robot with active pipe-diameter adaptability and automatic tractive force adjusting", Mechanism and Machine Theory, vol. 42, no. 12, pp. 1618-1631, 2007.

[4] D. Lee, J. Park, D. Hyun, G. Yook and H. Yang, "Novel mechanisms and simple locomotion strategies for an in-pipe robot that can inspect various pipe types", Mechanism and Machine Theory, vol. 56, pp. 52-68, 2012.

[5] P. Singh and G. Ananthasuresh, "A Compact and Compliant External PipeCrawling Robot", IEEE Trans. Robot., vol. 29, no. 1, pp. 251-260, 2013.

[6] J. Gao and G. Yan, "Locomotion Analysis of an Inchworm-Like Capsule Robot in the Intestinal Tract", IEEE Transactions on Biomedical Engineering, vol. 63, no. 2, pp. 300-310, 2016.

[7] R. Usamentiaga, J. Molleda, D. Garcia, J. Granda and J. Rendueles, "Temperature Measurement of Molten Pig Iron With Slag Characterization and Detection Using Infrared Computer Vision", IEEE Trans. Instrum. Meas., vol. 61, no. 5, pp. 1149-1159, 2012.

[8] P. Hansen, H. Alismail, P. Rander and B. Browning, "Visual mapping for natural gas pipe inspection", The International Journal of Robotics Research, vol. 34, no. 4-5, pp. 532-558, 2014.

[9] A. Kittawornrat and J. Zimmerman, "Toward a better understanding of pig behavior and pig welfare", Anim. Health. Res. Rev., vol. 12, no. 01, pp. 25-32, 2010.

[10] J. Deng, H. Shen, C. Xue, S. Liu and X. Wang, "Design and Development of Screw-Drive In-Pipe Robot", AMM, vol. 43, pp. 1-4, 2010.

[11] Z. Hu and E. Appleton, "Dynamic characteristics of a novel self-drive pipeline pig", IEEE Trans. Robot., vol. 21, no. 5, pp. 781-789, 2005.

[12] J. Yao, S. Gao, G. Jiang, T. Hill, H. Yu and D. Shao, "Screw theory based motion analysis for an inchworm-like climbing robot", Robotica, vol. 33, no. 08, pp. 1704-1717, 2014.

[13] Q. Yu, J. Yu, J. Wang and J. Liu ,"The Spiral Driven and Control Method Research of the Pipe Cleaning Robot", International Journal on Advances in Information Sciences and Service Sciences, vol. 5, no. 1, pp. 484-490, 2013.

[14] L. Liang, H. Peng, B. Chen, Y. Tang, S. Chen and Y. Xu, "Performance analysis and parameter optimization of an inner spiral in-pipe robot", Robotica, vol. 34, no. 02, pp. 361-382, 2014.

[15] P. LI, "Screw Drive In-pipe Robot with Axial and Circum-directional Inspection Ability", Journal of Mechanical Engineering, vol. 46, no. 21, pp. 19-28, 2010.

[16] K. Jang, H. Park and B. Kim, "Theoretical Velocity Analysis of Micro Robot Based on Crawling Locomotive Mechanism for Pipe Inspection Micro Robot", Transactions of the Korean Society of Mechanical Engineers A, vol. 32, no. 8, pp. 633-641, 2008.

[17] C. Lin, H. Gong, N. Nie, Q. Zen and L. Zhang, "Geometry design, threedimensional modeling and kinematic analysis of orthogonal fluctuating gear ratio face gear drive", Proceedings of the Institution of Mechanical Engineers, Part C: Journal of Mechanical Engineering Science, vol. 227, no. 4, pp. 779-793, 2012. 